\title{
Turbulence structure of polymer turbulent channel flow with and without macromolecular polymer structures
}

\begin{abstract}
The presence of macromolecular polymer structures in a fully developed turbulent channel flow has been shown to substantially increase the drag reduction compared to non-structured polymer flows. This study presents a detailed analysis of experimental data obtained using laser Doppler velocimetry (LDV) to develop insights into the effects of the presence of macromolecular polymer structures on the turbulence characteristics of a channel flow. It is argued that polymer structures could contribute to minimizing the interaction between the inner and outer regions of the flow, which, in turn, can contribute to the modification of the coherent structure of the turbulence.
\end{abstract}

\section{Introduction}

Most studies that focus on the effects of polymers on turbulence deal with fully developed flows in either circular pipes or rectangular channels (e.g., Rieschman and Tiederman 1975; Mizushina and Usui 1977; McComb and Rabie 1982; Willmarth et al. 1987; Luchik and Tiederman 1988; Harder and Tiederman 1991; Wei and Willmarth 1992, among others). The study of Rieschman and Tiederman (1975) showed that optimum efficiency is achieved when the polymers are located in the buffer region of the boundary layer. For low percentages of drag reduction, the effects of the addition of polymers on the mean velocity profile translates into an unchanged viscous sublayer and a shift of the logarithmic region towards higher velocities while remaining parallel

K. Kim · A. I. Sirviente $(\bowtie)$

Department of Naval Architecture and Marine Engineering, University of Michigan, Ann Arbor, MI 48109-2145, USA

E-mail: asirv@engin.umich.edu

Tel.: + 1-734-6479411

Fax: + 1-734-9368820 to the logarithmic layer of the corresponding Newtonian velocity profile. Large percentages of drag reduction seem to come associated with an increase in the slope of the logarithmic layer up to a maximum indicated by the maximum drag reduction asymptote (MDR; Virk 1975). Polymer flows show the peak of the streamwise velocity fluctuations at larger values of $y^{+}$than the corresponding Newtonian flow. However, the maximum value of the streamwise turbulence intensity differs substantially among studies. The normal turbulence intensities, on the other hand, consistently show attenuation in the buffer region. The Reynolds shear stress in polymer flows has been shown to decrease in the buffer region. The experiments of Willmarth et al. (1987) and Harder and Tiederman (1991) showed that a Reynolds stress defect could take place for drag reduction levels around $35 \%$ when using a polyethylene oxide (PEO) solution and a polyacrylamide (PAM) solution, respectively. A Reynolds stress defect implies that the sum of the viscous stress and the Reynolds stress is not equal to the stress calculated from the corresponding pressure gradient. Similar results have been confirmed recently by other studies (e.g., Warholic et al. 1999; Den Toonder et al. 1997; Ptasinski et al. 2003, etc.). The visualization studies of Donohue et al. (1972) reported an increase in the streak spacing of the coherent turbulent boundary layer in the presence of drag-reducing polymers and a significant decrease in the bursting rate. The real-time holography experiments of Achia and Thompson (1977) corroborated the latter findings, as did the studies of Berman (1986), Tiederman et al. (1985), and Luchik and Tiederman (1988), among others. Recently, some direct numerical simulations of drag reduction with polymer additives have questioned the theories of Lumley (1977) and de Gennes (1990), which consider that polymer drag reduction can be explained based on the modification of the small dissipative scales due to the additives. The studies of De Angelis et al. (2003) and Benzi et al. (2003) argue that the mechanism of drag reduction is mainly linked to the modification of the dynamics of the large scales. 
In the past, some consideration has been given to the differences and similarities between homogeneous and heterogeneous drag reduction in fully developed flows. However, the amount of work on heterogeneous drag reduction is very limited when compared to that on homogeneous drag reduction, where very dilute polymer solutions are either premixed in the flow of interest or else injected such that homogeneity of the resulting flow is ensured. Heterogeneous solutions are characterized by the presence of threads of concentrated polymer solutions. Heterogeneous drag reduction has been observed by Vleggaar and Tels (1973), Bewersdorff (1984), and Berman (1986), among others. These studies focused on the injection of highly concentrated polymer solutions into the centerline of pipe flows. The studies of Hoyer and Gyr $(1996,1998)$ concluded that the large levels of drag reduction attained by such heterogeneous polymer flows could be due to agglomerates of dissolved polymer molecules that translate into larger polymer concentrations close to the wall. The study of Vlachogiannis and Hanratty (2004) showed that wall injection of concentrated hydrolyzed polyacrylamide (HPAM) can achieve very large levels of drag reduction when polymer structures are present in the solutions prior to injection. Previous studies reported by the authors (e.g., Shen et al. 2003; Kim et al. 2004) address the comparison of homogeneous and heterogeneous polymer flows for the same flow characteristics and the same average polymer concentration at the channel test section when the polymer is injected at the wall. Those studies showed larger drag reduction for the heterogeneous polymer flow and that polymer structures, even if not present in the prepared solution, could be induced by the injection system and the mixing process that takes place in the channel. Those structures were mostly located in the neighborhood of the channel centerline. Kim et al. (2004) reported on the laser Doppler velocimetry measurements of the mean and the turbulent characteristics of both homogeneous and heterogeneous polymer flows in a fully developed turbulent channel flow. That study presented the distribution of the velocity fluctuations and the shear stress across the channel, and did not seem to indicate substantial differences in the region where the majority of the polymer structures are present. The purpose of this study is to conduct a detailed analysis of the higher order moments of turbulence along with the power spectra of the data, and the corresponding governing length scales of both heterogeneous and homogeneous flows. This study attempts to further establish the differences, if any, that the large macromolecular polymer structures might be inducing on the flow by congregating mostly along the channel centerline.

\section{Experimental apparatus and procedures}

This experimental study makes use of a recirculating water channel where the flow is driven by a $0.076 \mathrm{~m}^{3} / \mathrm{s}$ centrifugal pump into a $5.99-\mathrm{cm}$ wide by $59.94-\mathrm{cm}$ high and $6.35-\mathrm{m}$ long channel test section. The bulk velocity in the test section can be up to $2.11 \mathrm{~m} / \mathrm{s}$, leading to a Reynolds number based on the channel's width of up to $1.26 \times 10^{5}$. The measurement station is located $5.28 \mathrm{~m}$ (or 88.5 channel widths) downstream of the test section entrance and 84.4 channel widths from the injection slot. Twenty-one equally spaced pressure taps located on one of the test section walls monitor the pressure gradient. The polymer injection slots are located $22.86 \mathrm{~cm}$ (or 4.1 channel widths) downstream of the test section entrance on both sides of the channel. They are $0.25-\mathrm{cm}$ wide, $59.94-\mathrm{cm}$ high and are inclined at an angle of $25^{\circ}$ to the wall. A pneumatic system using compressed nitrogen drives the polymer solution from the 16-gallon pressure storage tanks to the injection slots. Flow meters near each injection slot are used to monitor the flow rate. Optical access to the measurement station for the LDV measurements is provided by slots $(2.54-\mathrm{cm}$ high by $15.24-\mathrm{cm}$ long) located on the side walls of the test section that are covered with $0.005-\mathrm{cm}$-thick, clear Mylar film. By adjusting the water levels in the two small water tanks on both sides of the channel, the pressure exerted on the Mylar film by the water in the tanks is forced to match the pressure exerted by the water in the test section. The channel water, polymer solution, and room temperatures are measured using type $\mathrm{K}$ thermocouples with $0.1{ }^{\circ} \mathrm{C}$ resolution. A detailed description of this experimental facility can be found in Kim et al. (2004).

\subsection{Experimental instrumentation}

Laser Doppler Velocimetry was used in this study to measure the velocity components and corresponding turbulence characteristics. The LDV system makes use of a 5-W Coherent Innova 70C argon-ion laser. The laser beam is split into three different color beam pairs and fiber optic cables were used to couple them with the custom-made, single, waterproof three-component LDV probe made by TSI Inc. In this study, only 2D measurements are reported. The beams are crossed at the same point and aligned with the receiving fiber. Each beam pair forms an effective measuring volume approximately $83 \mu \mathrm{m}$ in diameter with a length-todiameter ratio of approximately 2.5:1. The LDV system is operated in the coincidence mode and acousto-optic Bragg cells are used to shift the frequency of one beam in each pair. Scattered light is collected in the back-scatter mode, color filtered, and focused onto individual photomultipliers. The signals from the frequency downmixers are fed onto a TSI IFA 655 digital-burstcorrelator signal processor that determines and records the frequency of the Doppler bursts from all channels after band-pass-filtering the signals. The correlation processor is digitally interfaced to a Pentium II PC to process the signals. The water in the channel and the polymer solutions are seeded with titanium dioxide of rutile particles in crystalline form of $3 \mu \mathrm{m}$ in diameter. Measurements were taken at 80 locations between walls. 
The sampling rate was around $50 \mathrm{~Hz}$ in the near wall region and reached up to $600 \mathrm{~Hz}$ near the centerline. At each measuring position, up to 50,000 velocity triplets were collected. These data were then number averaged and refined. Realizations that were more than three standard deviations away from the mean on any component were removed. Corrections for velocity bias were performed with the transit time weight method. This correction is based on the inverse proportionality of the burst time to the magnitude of the velocity vector. The signals were also corrected for electronic noise, determined from the power spectrum of the corresponding fluctuating velocity. Corrections were also made to account for the size of the measuring volume; approximately three viscous lengths in these experiments. A detailed description of the indicated error corrections can be found in Kim et al. (2004). The data for both water and polymer flows have an uncertainly of $1 \%$ on mean velocity, less than $1 \%$ on normal stresses and $3 \%$ on shear stresses, and $6 \%$ on skewness and flatness. A more detailed description of the experimental setup and procedures can be found in Kim et al. (2004).

\subsection{Polymer solution preparation and experimental procedures}

In this study, a non-ionic polyacrylamide (Hyperfloc NF301, Hychem Inc., Tampa, FL, USA) is used as the drag reducing agent. The weight average $\left(M_{\mathrm{w}}\right)$ molecular weight and $z$ average radius of gyration $\left(R_{\mathrm{g}, z}\right)$ were measured by multi-angle laser light scattering. The results are $M_{\mathrm{w}}=7.5 \times 10^{6} \mathrm{~g} / \mathrm{mol}$ and $R_{\mathrm{g}, z}=170 \mathrm{~nm}$ as reported by Kim et al. (2004). Preparation of the polymer solution involves de-aeration of filtered tap water by heating it while stirring magnetically. After combining the granular polymer, isopropyl alcohol, and water, the solution requires both long-term hydration and gentle mixing. The mixing is done with a Nuova magnetic stirrer for the first ten minutes and then rolled by a Bellco Cell Production Roller Apparatus. A comprehensive study to assess the consistency of the polymer solution preparation procedures was performed and further details can be found in Sun Chee Fore et al. (2004). The polymer solution is injected through the slots in the channel walls for up to ten minutes to ensure that no polymer build-up takes place in the channel. Recirculation through the channel consequently degrades the polymer and injection is then re-started. The measurements reported in this study were made at a Reynolds number equal to $5.6 \times 10^{4}$, based on the channel width and the centerline velocity. During the experiments, the temperature was held constant at $22 \pm 1^{\circ} \mathrm{C}$.

\section{Results}

As mentioned earlier, this comparative study of the turbulence structure of polymer flows with and without macromolecular polymer structures follows the study of Kim et al. (2004). A brief summary of the mean velocity, normal and shear stresses measured for injection concentrations $\left(C_{\mathrm{i}}\right)$ of $10,000 \mathrm{ppm}$ (heterogeneous polymer flow) and 1,000 ppm (homogeneous polymer flow), and an average test section concentration $\left(C_{\mathrm{h}}\right)$ of $14 \mathrm{ppm}$ presented in Kim et al. (2004), are presented herein. The percentage of drag reduction corresponding to $C_{\mathrm{i}}=10,000$ and $1,000 \mathrm{ppm}$ are 39 and $13.1 \%$, respectively. The shear velocity $u_{\tau}=\left(\tau_{\mathrm{w}} / \rho\right)^{1 / 2}$, where $\tau_{\mathrm{w}}$ is the wall shear stress and $\rho$ is the density of water, corresponding to the Newtonian, heterogeneous, and homogeneous polymer flows are $0.038,0.029$, and $0.035 \mathrm{~m} / \mathrm{s}$, respectively, which correspond to $R e_{\tau}$ (based on $u_{\tau}$, half channel width, and water viscosity at $22^{\circ} \mathrm{C}$ ) of 1,190 , 908, and 1,096, respectively. The results presented in Kim et al. (2004) showed that the case corresponding to higher injection concentrations displayed a change in slope in the logarithmic region of the streamwise mean velocity component, while for the low injection concentrations, the logarithmic law experienced a shift upwards. The maximum $u^{+}$was moved away from the wall in both polymer flows with respect to the Newtonian flow. The shift was more important for the heterogeneous polymer flow, for which there are important changes not only close to the wall, but also across the channel. The normal and Reynolds stresses are significantly reduced across the channel for the heterogeneous polymer flow, while the changes with respect to the Newtonian flow for the homogeneous polymer flow only take place in the region up to roughly $y^{+}=100$. The presence of macromolecular polymer structures was shown to concentrate mostly in the neighborhood of the channel centerline via flow visualization, turbidity, and birefringence measurements. The mechanism by which a larger concentration of polymer structures in the neighborhood of the channel centerline can translate to a larger reduction of wall shear stress in a turbulent channel flow was not clear from the results presented regarding mean velocity components, as well as normal and Reynolds stresses. The purpose of this study was to further probe the data to gather more knowledge regarding the turbulence structure of the flows with and without polymer structures to be able to shed more light on the phenomena of heterogeneous drag reduction.

\subsection{Further analysis of the turbulence structure}

A reduction in the turbulent intensity in the $y$ direction and also a lower contribution to turbulent momentum from the velocity fluctuations (shown by the distribution of the correlation coefficient as presented by Kim et al. (2004)) can be associated with the reduction of the Reynolds stress observed due to the presence of polymers in the flow. To further investigate this, the joint probability density function of $u v^{+}$as a function of $u^{+}$ and $v^{+}$for various locations across the channel was computed and is presented in Figs. 1, 2, and 3 for 

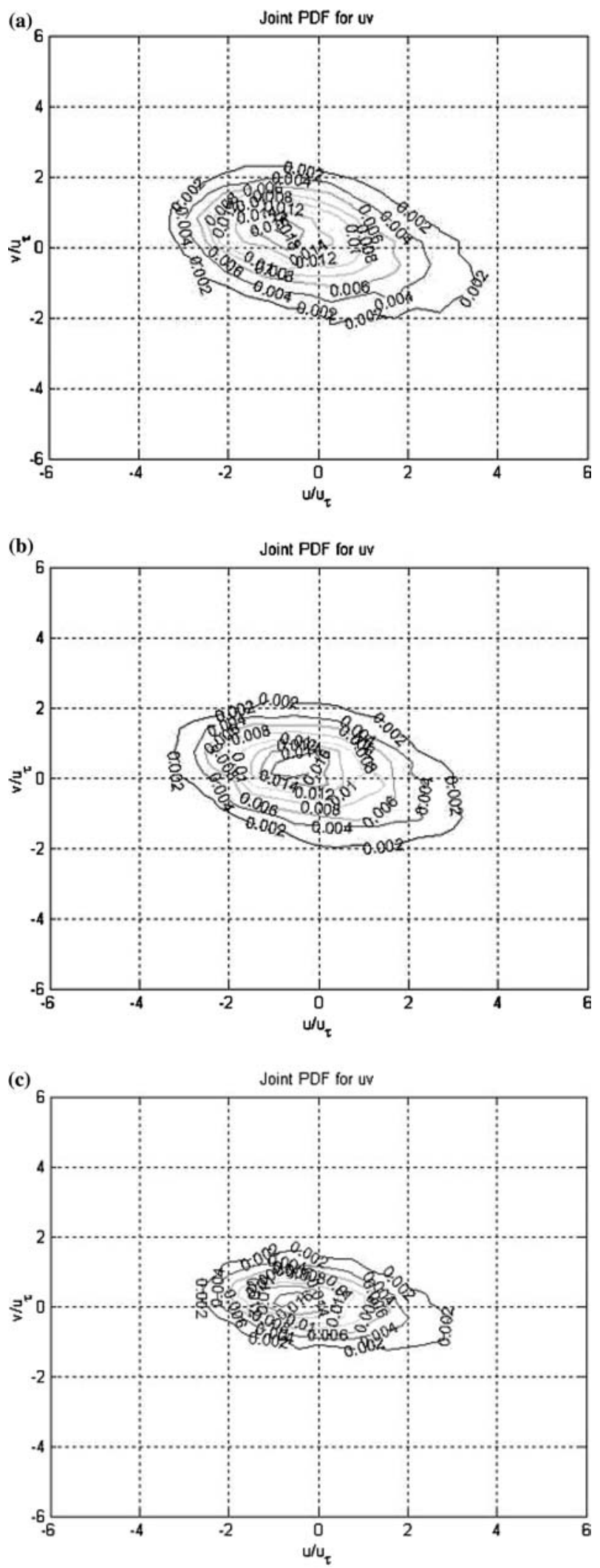

Fig. 1a-c Joint probability density function of steamwise and normal velocity fluctuations at $y^{+}=400$ for a Newtonian flow, b polymer flow with $C_{\mathrm{i}}=1,000 \mathrm{ppm}$, and c polymer flow with $C_{\mathrm{i}}=10,000 \mathrm{ppm}$
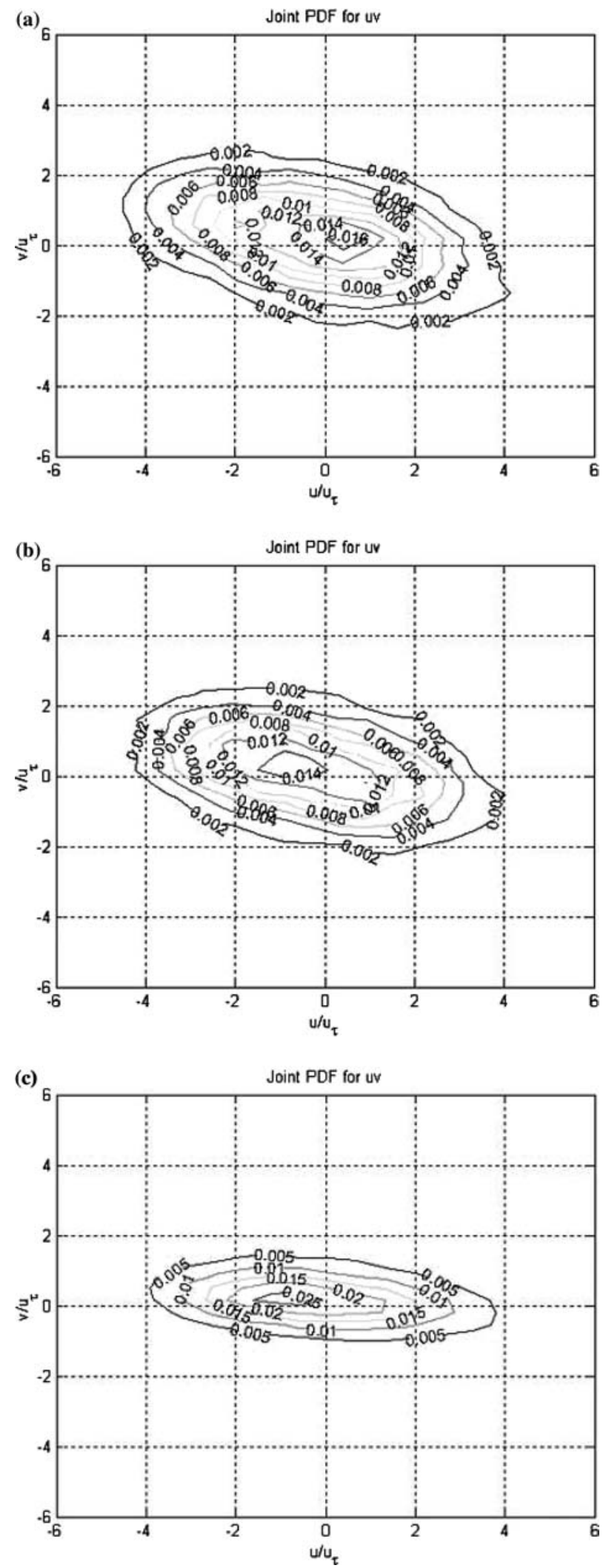

Fig. 2a-c Joint probability density function of steamwise and normal velocity fluctuations at $y^{+}=100$ for a Newtonian flow, b polymer flow with $C_{\mathrm{i}}=1,000 \mathrm{ppm}$, and c polymer flow with $C_{\mathrm{i}}=10,000 \mathrm{ppm}$ 

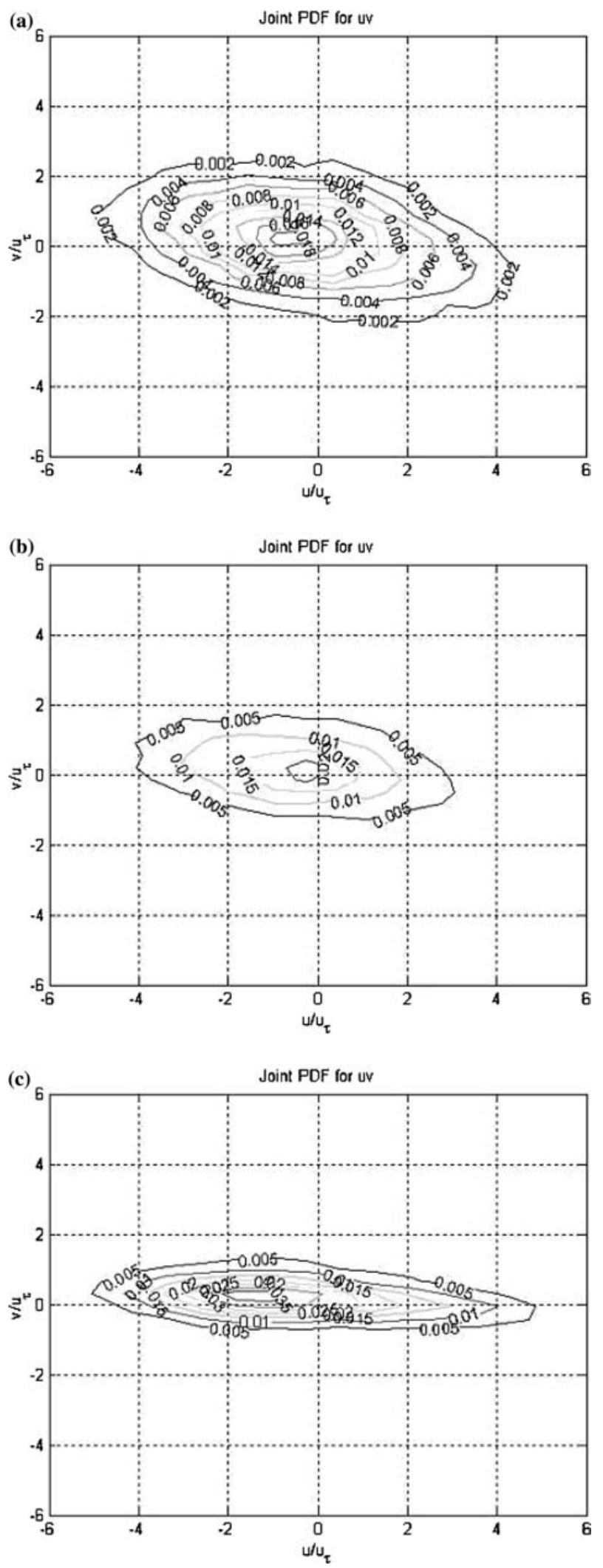

Fig. 3a-c Joint probability density function of steamwise and normal velocity fluctuations at $y^{+}=50$ for a Newtonian flow, b polymer flow with $C_{\mathrm{i}}=1,000 \mathrm{ppm}$, and c polymer flow with $C_{\mathrm{i}}=10,000 \mathrm{ppm}$ $y^{+}=400,100$, and 50, respectively. The reduction in the rms of the normal velocity fluctuation in the polymer cases is made obvious by the important anisotropy of the fluctuations shown in the figures corresponding to the joint probability density function. In all cases, and as expected, the probability of events from quadrants II and IV is higher than for quadrants I and III. Figure 1a, b shows that, at $y^{+} \sim 400$, the results for $C_{\mathrm{i}}=1,000 \mathrm{ppm}$ and water are practically identical, unlike for $C_{\mathrm{i}}=10,000 \mathrm{ppm}$. In the heterogeneous polymer flow, the magnitude of both streamwise and normal velocity fluctuations have decreased, and there is also a decreased occurrence of realizations in quadrants I and III. Similar behavior is observed further away from the wall and closer to the centerline. Figure 2, corresponding to a location of $y^{+} \sim 100$, shows once again quite similar results between water and the homogeneous polymer flow, despite a small reduction in the $v$ fluctuations in the latter case. On the other hand, the changes observed for the heterogeneous polymer flow are quite important, with an important reduction in the $v$ fluctuations. In this case, the principal axes for the Reynolds stress tensor have rotated significantly towards the laboratory axes, displaying an angle of $-5.37^{\circ}$, while it is $-17.1^{\circ}$ for the Newtonian flow. By $y^{+} \sim 50$ (Fig. 3), the principal axes become practically aligned with the laboratory axes in the case of the heterogeneous flow, for which there is a clear increase of the $u$ fluctuations, while the $v$ fluctuations are being significantly damped. In this case, the principal stress axes showed an angle of $-2.68^{\circ}$ with the laboratory axes, in comparison to $-12.6^{\circ}$ corresponding to the Newtonian flow. The homogeneous flow at the same $y$ location also showed that the principal axes become more parallel to the flow direction $\left(-8.02^{\circ}\right)$, while no significant changes in the magnitudes of the fluctuations are appreciable compared to the Newtonian flow. The changes in orientation of the principal axes of the Reynolds stress tensors, with respect to the laboratory axes, translate into a smaller magnitude of Reynolds stresses in the drag-reduced flows and a reduction in the correlation coefficient. The presence of the polymer structures seems to not only enhance the effects seen with homogeneous polymer flows near the wall, but, more importantly, to maintain those effects all across the channel. Figure 4 a shows the distribution of the angle of the principal shear stress axes computed from the mathematical formulation of the shear stress in a rotated coordinate system (Hinze 1975):

$u v^{*}=\left(v^{2}-u^{2}\right) \sin \alpha \cos \alpha+u v\left(\cos ^{2} \alpha-\sin ^{2} \alpha\right)$

after computing the angle that maximizes the principal shear stress, where $u v^{*}$ is the principle shear stress. The reduction of the angle of the principal shear stress when polymers are present is remarkable in comparison to the Newtonian flow. This reduction is particularly important within the near wall region for homogeneous flows $\left(y^{+}<100\right)$ and all through the 
channel for heterogeneous flows. Figure $4 \mathrm{~b}$ shows the corresponding distribution of the maximum shear stress $\left(u v^{*}\right)$. In the near wall region, the presence of the polymers induces an increase of the principal shear stress with respect to the Newtonian flow. In the homogeneous flow, this increase seems to take place in the region $y^{+}<100$, while in the heterogeneous flow, it happens in $y^{+}<300$. Consequently, the region where the polymer molecules can be stretched is substantially increased by the presence of the polymer structures.
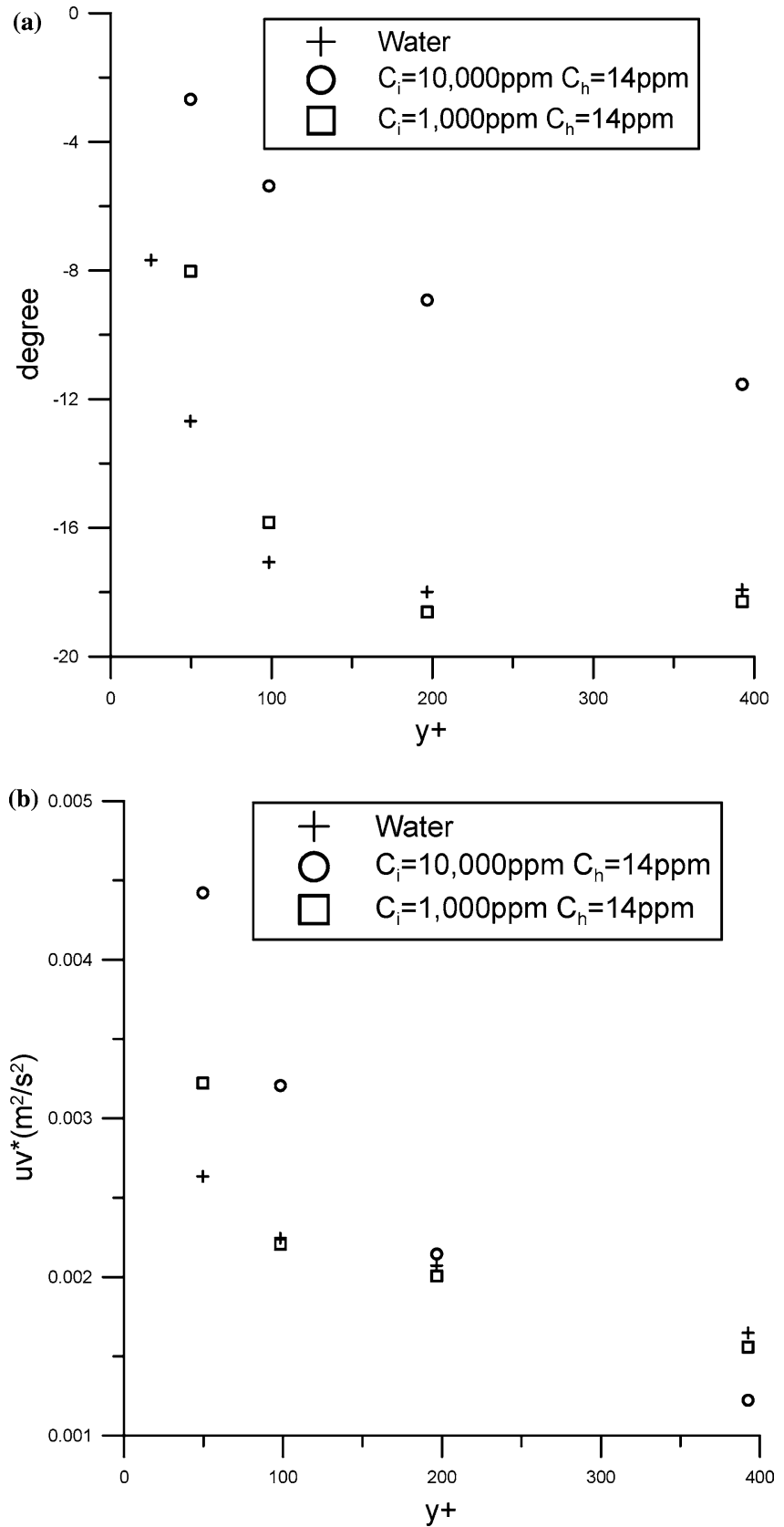

Fig. 4 a Distribution of the angle of the principal shear stress axes and $\mathbf{b}$ distribution of the principle shear stress for the Newtonian and both polymer flows

\subsubsection{Turbulence production}

Turbulent kinetic energy production, in general, is maximum close to the wall due to the strong mean shear and large Reynolds stresses in that region. Figure 5a shows the production term corresponding to the normal stress $u u$ for water, as well as both polymer flows, and Fig. $5 \mathrm{~b}$ shows the production of shear stress $u v$. The mean steamwise velocity gradient was determined by differentiating the best polynomial fit to the measured mean velocity. The results agreed to within $5 \%$ of those obtained from differentiating the experimental data directly. The data corresponding to the lower injection
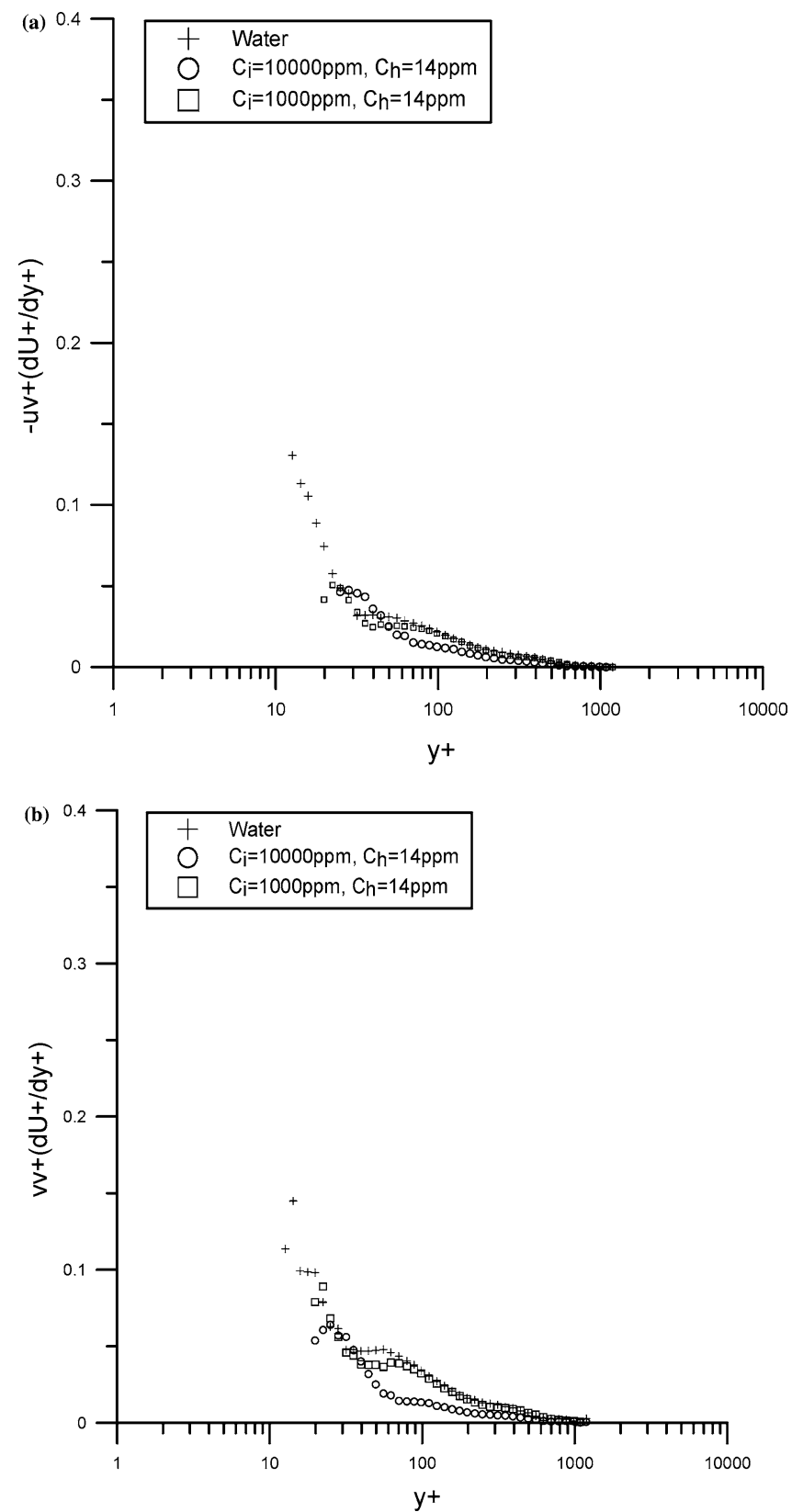

Fig. 5a, b Production of a Reynolds normal stress $\left(u^{2}\right)$ and b Reynolds shear stress for the Newtonian and both polymer flows 
concentration show a remarkable similarity to that of water from the centerline up to approximately $y^{+} \sim 60$ when production is decreased; in fact, the maximum attained is $\sim 0.05$ at around $y^{+} \sim 25$. In contrast, the data corresponding to the highest injection concentration show agreement with that from water without injection, from the channel centerline up to approximately $y^{+} \sim 200$. The maximum production seems to take place around $y^{+} \sim 30$, roughly with the same magnitude as that measured for the homogeneous flow. The streamwise velocity fluctuations for the structured polymer flow are lower than those corresponding to water or the homogeneous polymer flow from the centerline up to approximately $y^{+} \sim 150$ and for $y^{+}<25$. At $y^{+} \sim 35$, the streamwise velocity fluctuations corresponding to the structured polymer solution become a maximum. The normal velocity fluctuations are consistently lower than water all across the channel.

The results presented in Fig. 5b for the production of $u v$ show identical distributions up to $y^{+} \sim 60$ for both water and homogeneous polymer flows. From there onto the wall, the values of shear stress production measured for the homogenous polymer flow are lower than those corresponding to the water flow. An important reduction of the Reynolds shear stress with respect to water takes place for the homogeneous polymer flow in $y^{+}<150$. The maximum turbulent stress takes place around $y^{+} \sim 150$. The results obtained for the production of $u v$ when polymer structures are present are lower than those corresponding to water and the homogeneous polymer solution results, almost consistently for $y^{+}<500$. The data corresponding to both homogeneous and heterogeneous polymer distributions become comparable in the region $30<y^{+}<40$, after which, and closer to the wall, the production of $u v$ becomes much smaller for the case with polymer structures.

\subsubsection{Higher order moments of the fluctuating velocity components}

The skewness and kurtosis, or flatness, corresponding to the streamwise and normal velocity fluctuations are shown in Figs. 6a, b, and 7a, b, respectively. These quantities represent the main characteristics of the corresponding probability density distributions. The skewness, or third moment, gives an indication of the symmetricity characteristics of the corresponding probability density function. The fourth moment, or flatness, gives an indication of how large the probability density function is in the tails of the distribution. They are defined as $S(u)=u^{3} /\left(u^{2}\right)^{3 / 2}$ and $F(u)=u^{4} /\left(u^{2}\right)^{2}$, respectively, for the streamwise velocity fluctuation and similarly for the other velocity components. Fluctuations with Gaussian distributions have zero skewness and a value of approximately three for kurtosis. As it can be seen from Figs. 6 and 7, in the near wall region, both high order moments differ significantly from the values corresponding to a Gaussian distribution, and more so for the normal velocity fluctuations. The data shown in Fig. 6a for the skewness of the axial velocity fluctuations shows the expected trend for the water data. The data agrees with the results reported by Wei (1987) for channel flows with Reynolds numbers in the range $2,970<\operatorname{Re}<39,582$. Figure 6 a also shows comparable magnitudes for the lower polymer injection concentration case with respect to the results from water, except for the region $20<y^{+}<100$, where the skewness of the polymer flow is lower than that of water, and $y^{+}>500$, where it is larger. The region $20<y^{+}<100$ coincides with the portion of the channel were the magnitude of the streamwise velocity fluctuations of the drag-reducing flow are significantly larger than those corresponding to the Newtonian flow. On the other hand, the skewness corresponding to the normal velocity fluctuations shown in Fig. 6b show consistent Gaussian-like results for the homogeneous polymer flow and the Newtonian flow from roughly around the same $y^{+}$location, where the maximum of $v^{+}$takes place; that is, $y^{+} \sim 100$, onto $y^{+} \sim 500$ from where $S(v)$ becomes slightly larger than zero in both flows. The largest injection concentration case shows initially larger values of the skewness of the streamwise velocity fluctuation with respect to the water data up to $y^{+}=30$ and lower afterwards up to $y^{+} \sim 500$. The increase of the skewness is due to the decrease in the same region of the mean velocity with respect to the Newtonian flow. For $y^{+}>500$, the values of $S(u)$ for both polymer flows, that is, with and without polymer structures, are comparable and slightly larger than those corresponding to water. The region of negative skewness is largest for the flow with macromolecular polymer structures present, which will come associated with the reduction in mean velocity experienced by the flow in that region. Large negative values of $S(u)$ might be associated with more large-amplitude negative streamwise velocity fluctuations than positive ones. For dilute homogeneous polymer flows, Luchik and Tiederman (1988) showed a reduction of ejections in the near wall region. Taking this into account, the larger region of negative skewness, if associated with less ejections, should have a much larger amplitude than otherwise found in Newtonian flows. The skewness of the normal velocity fluctuations presented in Fig. 6b shows Gaussian-distributed fluctuations for both polymer flows from roughly the location where the maximum of $v^{+}$takes place, $y^{+} \sim 200$, and all the way to the centerline. In the near wall region, $S(v)$ of the polymer flow with the highest concentration is consistently much lower than $S(v)$ for both Newtonian and homogeneous polymer flows.

The flatness of the streamwise velocity fluctuation (Fig. 7a) shows lower values than a Gaussian distribution in the region $10<y^{+}<50$ for both polymer flows and also for the Newtonian flow. The corresponding flatness for both polymer flows show lower values than those of water up to $y^{+}=50$, and comparable magnitudes thereafter. The kurtosis corresponding to the normal velocity fluctuation shown in Fig. $7 \mathrm{~b}$ show 
consistent Gaussian-like results after $y^{+}=30$ for the Newtonian and the low-polymer-concentration flows. The changes in $F(v)$ for the structured polymer flow take place over a larger region, $y^{+}<200$.

\subsubsection{Turbulent length scales}

To get a better understanding of the effect of the polymer molecules and structures on the flow, the integral and Kolmogorov length scales were computed. Following the approach of Warhaft and Lumley (1978) and Chambers and Antonia (1984), the integral length scale,
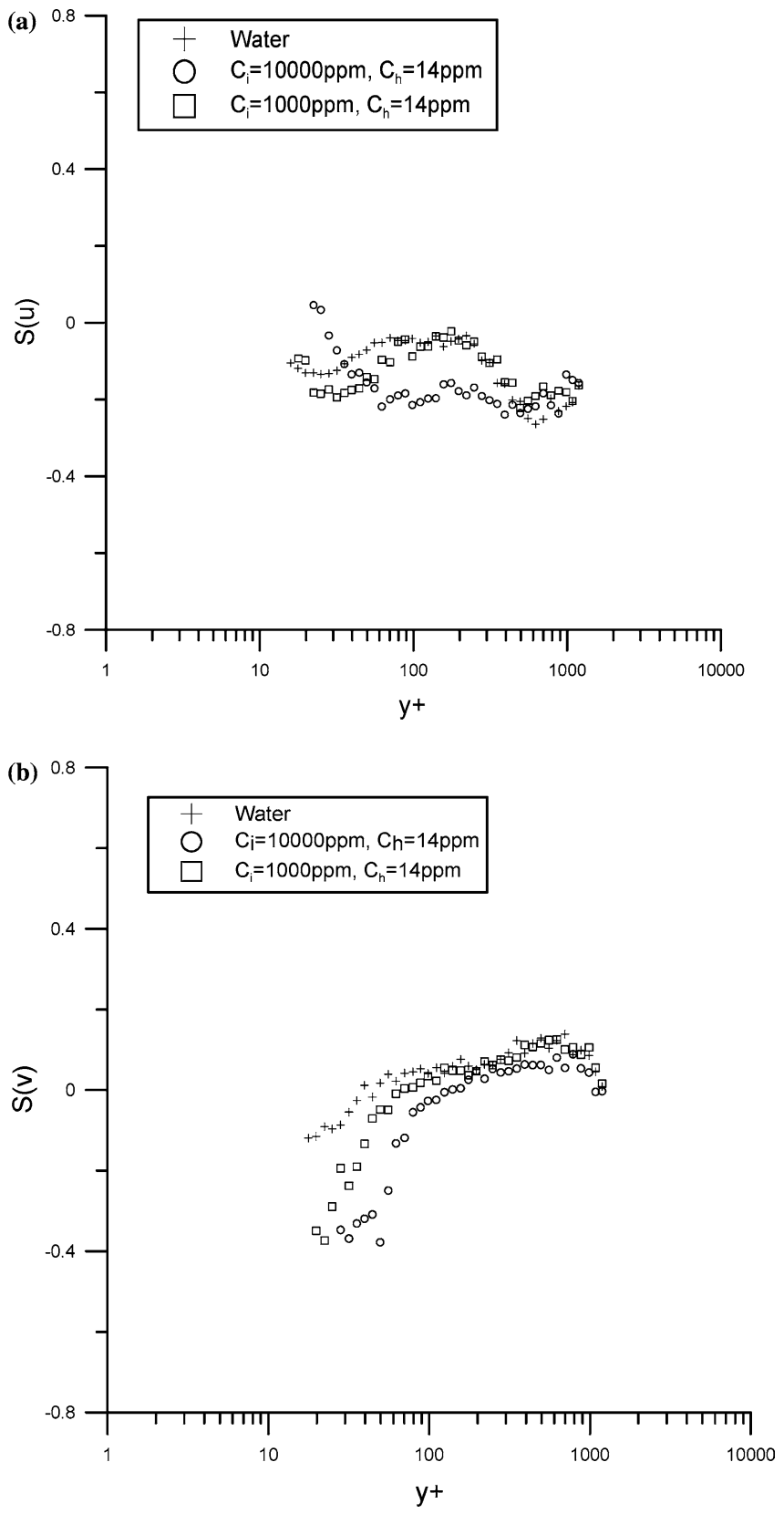

Fig. 6a, b Skewness of the a streamwise and b normal velocity fluctuations for the Newtonian and both polymer flows $\ell$, was determined by invoking Taylor's frozen turbulence hypothesis as the inverse of the wave number corresponding to the peak of the energy spectra, determined by multiplying the power spectra by the corresponding wave number. The integral length scale represents the size of the strongest eddies in the flow. The results corresponding to the distribution of the integral length scale across the channel for both polymer flows and the Newtonian flow are shown in Fig. 8a. The results indicate that the integral scales for the larger injection concentration polymer flow are larger than those corresponding to the Newtonian flow and the homogeneous polymer flow. Important discrepancies
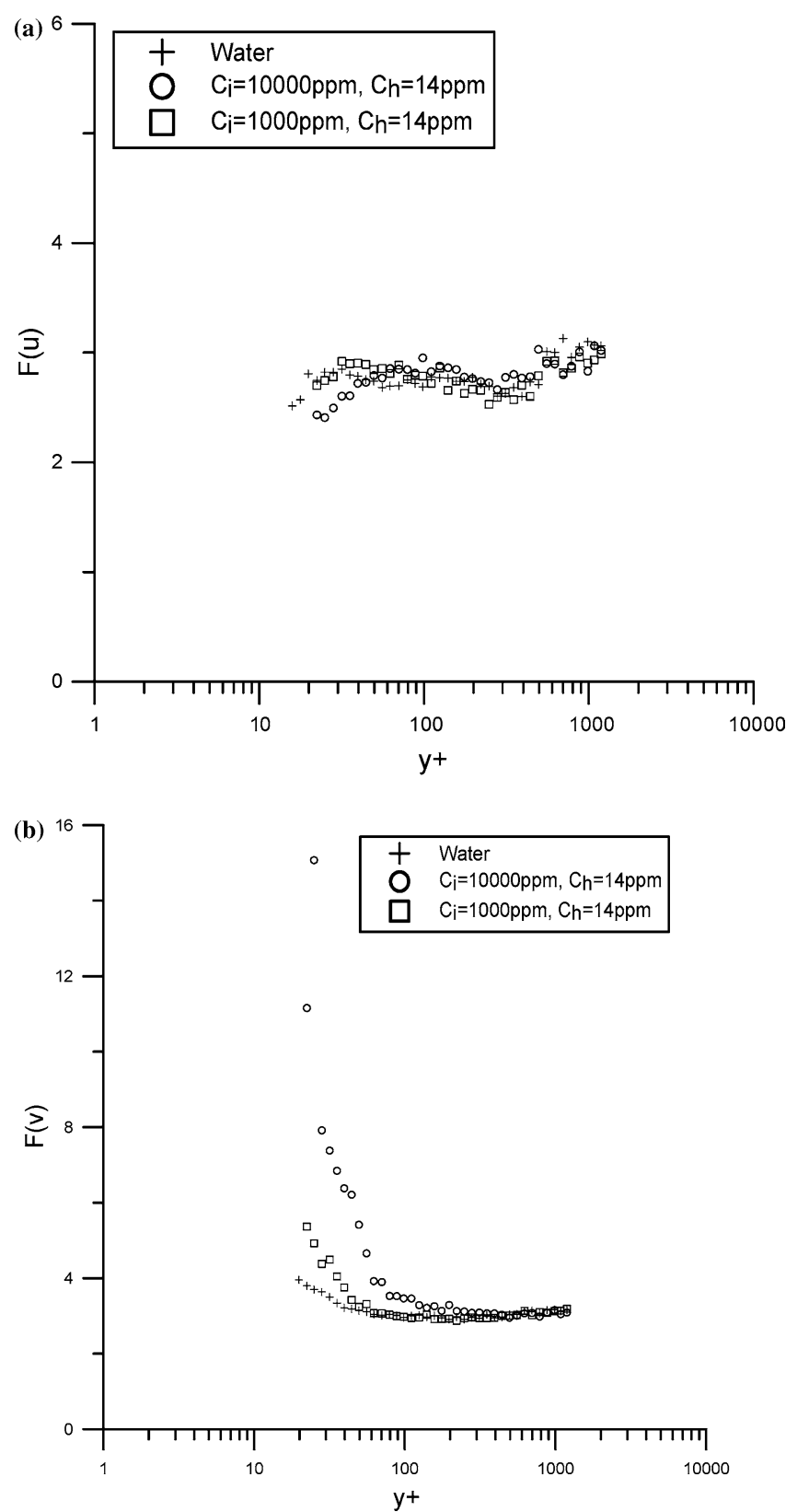

Fig. 7a, b Flatness of the a streamwise and b normal velocity fluctuations for the Newtonian and both polymer flows 
take place close to the wall, where the heterogeneous flow integral length scale is about 1.8 and 3.4 times larger than those of the homogeneous and Newtonian flows, respectively, at $y^{+} \sim 50$. In the region closer to the channel centerline, where the bulk of the polymer structures concentrate there, are also significant differences indicating that, in that region, the strongest eddies in the flow are larger for the heterogeneous polymer flow. The sizes of the integral length scale close to the centerline seem to be comparable to the sizes of the polymer structures as inferred from the flow visualizations performed as part of this study. Qualitatively, this will seem to indicate that the polymer structures are large enough to interact with the larger eddies present in the flow and possibly affect their motion.

To test the behavior of the smallest eddies in the flow, the Kolmogorov length scale was computed also for both polymer flows and the Newtonian flow at various locations across the channel. The Kolmogorov length scale is defined as $\eta=\left(v^{3} / \varepsilon\right)^{1 / 4}$, where $\epsilon$ is the dissipation rate computed based on the scaling law $\varepsilon=\left(u^{2}\right)^{3 / 2} / l$, where $\ell$ is the turbulence integral scale calculated as indicated above.

The results corresponding to the Kolmogorov length scale distribution across the channel are presented in Fig. 8 b. From the results, it can be observed that the size of the smallest eddies has increased over that of the corresponding Newtonian flow for both polymer flows. At $y^{+} \sim 50$, the Kolmogorov length scale is 1.2 and 1.1 times that corresponding to the Newtonian flow. The conclusions reached for homogeneous polymer flows in the past (Hinch 1977; Koskie and Tiederman 1991, etc.) regarding the damping of the smallest eddies by the presence of the polymer are corroborated by the results shown herein for the homogeneous polymer flow. In the case of the heterogeneous polymer flow, the latter conclusion seems to be aggravated; that is, the sizes of the smallest eddies in this flow are much larger than in the corresponding homogeneous polymer flow. The main difference between both homogeneous and heterogeneous polymer flows does not take place only in the near wall region, but also, important changes to the eddy sizes can be appreciated closer to the channel's centerline.

\section{Discussion}

The ability of a turbulent flow to stretch a polymer and consequently modify the viscosity resulting in the damping of small eddies and the thickening of the viscous sublayer has been argued by Lumley (1977) to explain the phenomenon of polymer drag reduction. On the other hand, de Gennes (1990) argued that the elastic characteristics of the polymers are the main mechanism behind polymer drag reduction. Both qualitative theories seem to be able to possibly explain some aspects of polymer drag reduction reported by different experimentalists in the past. A complete quantitative descrip- tion of the wall turbulence dynamics and its modification due to the presence of the polymers is still not available.

From the results presented herein and in Kim et al. (2004), it can be observed that the well documented effects of polymers in turbulent flows take place in both homogeneous and heterogeneous turbulent channel flows for the same average concentration at the test section. Nonetheless, the fact that the level of drag reduction for the heterogeneous polymer flow is more than double than that obtained for the homogeneous polymer flow while the macromolecular polymer struc-
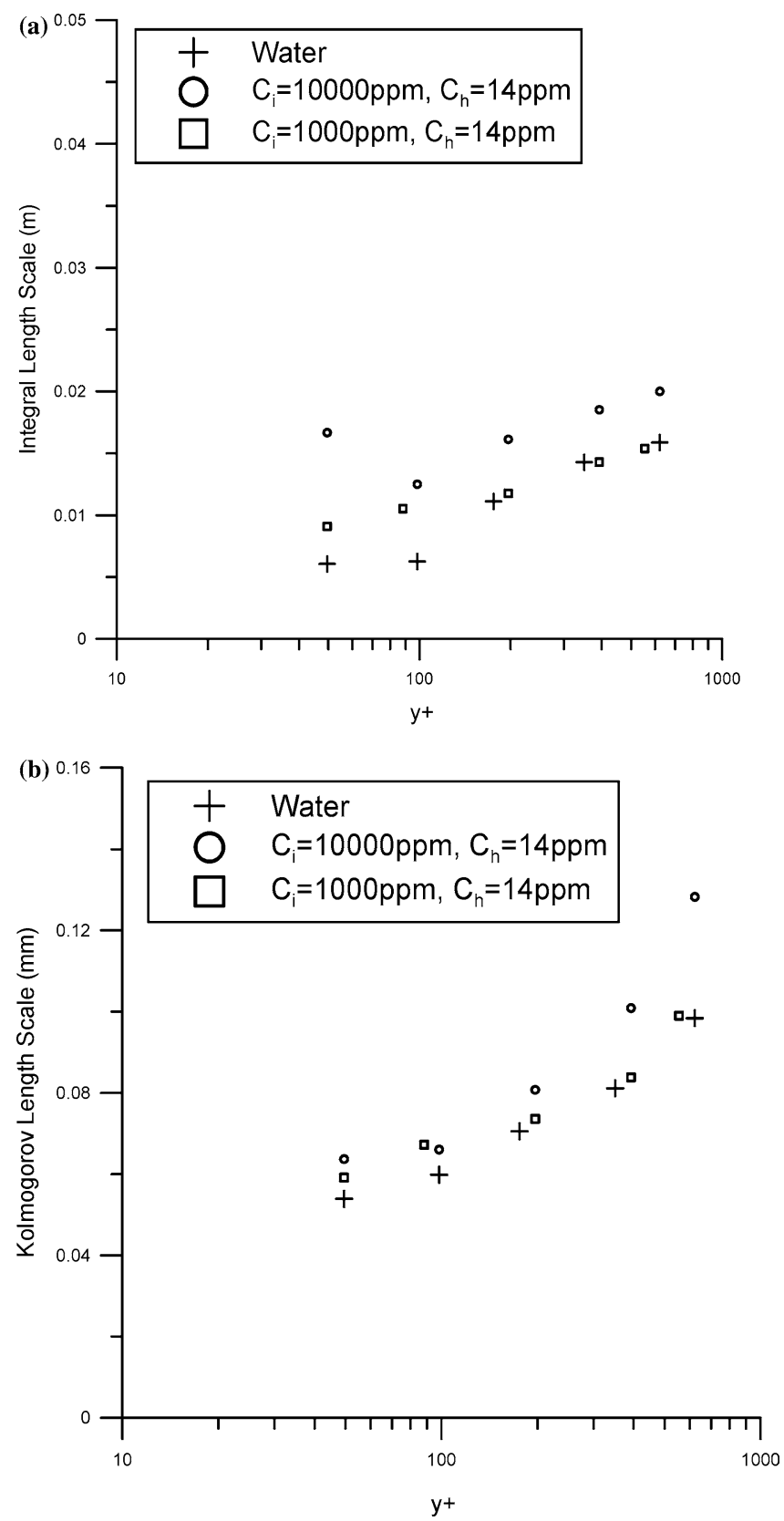

Fig. 8a, b Distributions of the a integral length scale and $\mathbf{b}$ the Kolmogorov length scale across the channel for the Newtonian flow and both polymer flows 
tures are mostly found to stay in the neighborhood of the centerline of the channel seems a surprising result if, indeed, polymer drag reduction is exclusively a near wall phenomenon.

In accordance to Lumley's theory, the uncoiled polymer molecules, which will tend to orient in the same direction of the flow, will induce an increased viscosity near the wall, caused by elongational deformation of the polymer molecules, which will translate into the characteristic turbulence anisotropy that appears in polymer flows and that is shown here for both homogeneous and heterogeneous polymer flows. That is, the extended polymer molecules inhibit the amplitude of the normal velocity fluctuations significantly. The relaxation of the polymers takes place by transfer of some of the mean and turbulent kinetic energy into elastic energy to the polymers that, in turn, translate into the uncoiling of the molecules, which results in inhibiting the transfer of energy among the streamwise and normal velocity fluctuations (Ptasinski et al. 2003). The ability of the turbulent flow to relax the polymers has been shown herein by demonstrating that important extensional motions exist for both homogeneous and heterogeneous polymer flows. The region where polymers can potentially be stretched by the turbulent flow seems to be larger for the heterogeneous flow. Also, when polymers are present, the most energetic velocity fluctuations take place in the flow direction. Consequently, it is expected that there will be more streamwise stretching of the vorticity field in the inner region of the flow when polymer is present; that, is the coherent structures in this region will be oriented more parallel to the mean flow. This conclusion agrees with the results shown by particle image velocimetry measurements shown in the literature, as well as by direct numerical simulations (e.g., Warholic 1997; Sureshkumar et al. 1997; Dimitropoulos et al. 2001, etc.)

In the near wall region of a Newtonian flow, the turbulence process is dominated by the production of dissipative scales, or small-scale vortices, by mean shear. It has been conjectured that the large extensional viscosity of the polymer flows in the near wall region is able to influence this process by damping the production of the dissipative scales. This translates into an increase of the Kolmogorov length scale for the drag-reduced flow. This has also been shown in this study for both homogeneous and heterogeneous polymer flows from the values of the Kolmogorov length scale. The changes for the homogeneous polymer flow are relatively small with respect to the Newtonian flow, but those found in the heterogeneous flow are quite substantial. It is conceivable that the bursting period, as has been shown by some investigators in the past for homogeneous flows (Luchik and Tiederman 1988), will decrease due to the very large elongational viscosity that will minimize the instabilities leading to bursting.

In the near wall region, the main difference between the homogeneous and heterogeneous polymer flows studied herein seems to be that small and sporadic polymer structures can be seen for the heterogeneous polymer flow. In the outer flow, the differences are substantial, with the presence of the larger bulk of polymer structures. It seems plausible to speculate that polymer structures could respond to a turbulent flow as a combination of effects by increasing the elongational viscosity in regions of strong extensional flow, but also by displaying elastic behavior, especially at high frequencies. This combination of effects (or theories, that is Lumley's and de Gennes') could result in an enhanced damping of the normal velocity fluctuations. Larger scale turbulence structures will be generated and smaller scales than the structures will be damped. The polymer structures might demonstrate a better ability to block the exchange of flow between the inner and outer regions of the flow and, as a consequence, the interaction of the dominant structures in both regions could be severely hindered. The trends shown by the length scales in the near wall region agree with these conclusions. This blocking effect will take place at certain intervals in time, during which, the polymer will be effective. As it degrades, this phenomenon will be relaxed and the entrainment of polymer from the outer region will then take place to further sustain drag reduction. This process of blocking the interaction between the inner and outer regions of the flow resembles the shear-sheltering mechanism discussed by Hunt and Durbin (1999) and also by Ptasinski et al. (2003) for homogeneous dilute polymer flows.

\section{Conclusions}

In this study, the effects of the presence of macromolecular polymer structures on the turbulence structure of a channel flow are discussed. It is argued that the extensional motions in the flow could not only potentially stretch the polymers, but also elastically force the polymer structures, contributing to a more significant damping of the normal velocity fluctuations. So long as the polymer molecules are non-degraded, the consequent effect could be a fluctuating minimization of the interaction between the inner and outer flows. This blocking effect induced by the polymers can potentially be enhanced by the presence of polymer structures and, consequently, it contributes to the modification of the coherent structure of the turbulence near the wall by orienting and stretching the vortical structures in the near wall region in the direction parallel to the mean flow. It also contributes to the damping of the smaller scales, which results in the increase of the Kolmogorov length scale in that region. The intermittency of the blocking mechanism imposed by the polymer molecules and enhanced by the polymer structures could allow for the feeding of polymer from the outer flow to the inner region of the flow. It is not obvious, based on these results, whether the bulk of polymer structures present in the channel's centerline could force the flow by modifying the larger turbulent structures. 


\section{References}

Achia BU, Thompson DW (1977) Structure of the turbulent boundary in drag-reducing pipe flow. J Fluid Mech 81:439464

Benzi R, De Angelis E, Govindarajan R, Procaccia I (2003) Shell model for drag reduction with polymer additives in homogeneous turbulence. Phys Rev E 68:016308-1-016308-10

Berman NS (1986) Velocity fluctuations in non-homogeneous drag reduction. Chem Eng Commun 42:37-51

Bewersdorff HW (1984) Effect of a centrally injected polymer thread in turbulent pipe flows. In: Sellin RHJ, Moses RT (eds) Drag reduction in fluid flows. Ellis Horwood, Chichester, UK

Chambers AJ, Antonia RA (1984) Atmospheric estimates of power-law exponent-mu and exponent-mu-theta. Boundary Layer Meteorol 28:343-352

De Angelis E, Casciola CM, L'vov VS, Piva R (2003) Drag reduction by polymers in turbulent channel flows: energy redistribution between invariant empirical modes. Phys Rev E 67:056312-1-056312-11

de Gennes PG (1990) Introduction to polymer dynamics. Cambridge University Press, Cambridge

Den Toonder JMM, Hulsen MA, Kuiken GDC, Nieuwstadt FTM (1997) Drag reduction by polymer additives in a turbulent pipe flow: laboratory and numerical experiments. J Fluid Mech 337:193-231

Dimitropoulos CD, Sureshkumar R, Beris AN, Handler RA (2001) Budgets of Reynolds stress, kinetic energy and streamwise enstrophy in viscoelastic turbulent channel flow. Phys Fluids 13(4): 1016-1027

Donohue GL, Tiederman WG, Reischman MM (1972) Flow visualization of the near-wall region in a drag-reducing channel flow. J Fluid Mech 56:559-575

Harder KJ, Tiederman WG (1991) Drag reduction and turbulent structure in 2-dimensional channel flows. Philos Trans R Soc Lond Ser A-Math Phys Eng Sci 336:19-34

Hinch EJ (1977) Mechanical models of dilute polymer solutions in strong flows. Phys Fluids 20:S22-S30

Hinze JO (1975) Turbulence. McGraw-Hill, New York, p 327

Hoyer KW, Gyr A (1996) Turbulent velocity field in heterogeneously drag reduced pipe flow. J Non-Newtonian Fluid Mech 65:221-240

Hoyer KW, Gyr A (1998) Heterogeneous drag reduction concepts and consequences. J Fluids Eng 120:818-823

Hunt JCR, Durbin PA (1999) Perturbed vortical layers and shear sheltering. Fluid Dyn Res 24:375-404

Kim K, Islam MT, Shen X, Sirviente AI, Solomon MJ (2004) Effect of macromolecular polymer structures on drag reduction in a turbulent channel flow. Phys Fluids 16(11):4150-4162

Koskie JE, Tiederman WG (1991) Turbulence structure and polymer drag reduction in adverse pressure gradient boundary layers. Technical report PME-FM-91-3, Purdue University, West Lafayette, Indiana

Luchik TS, Tiederman WG (1988) Turbulent structure in lowconcentration drag-reducing channel flows. J Fluid Mech 190:241-263

Lumley JL (1977) Drag reduction in two phase and polymer flows. Phys Fluids 20:S64-S71
McComb WD, Rabie LH (1982) Local drag reduction due to injection of polymer solutions into turbulent flow in a pipe. AICHE J 28:547-557

Mizushina T, Usui H (1977) Reduction of eddy diffusion for momentum and heat in viscoelastic fluid flow in a circular tube. Phys Fluids 20:S100-S108

Ptasinski PK, Nieuwstadt FTM, van den Brule BHAA, Hulsen MA (2001) Experiments in turbulent pipe flow with polymer additives at maximum drag reduction. Flow Turbulence Combust 66:159-182

Ptasinski PK, Boersma BJ, Nieuwstadt FTM, Hulsen MA, van den Brule BHAA, Hunt JCR (2003) Turbulent channel flow near maximum drag reduction: simulations, experiments and mechanisms. J Fluid Mech 490:251-291

Rieschman MM, Tiederman WG (1975) Laser-Doppler anemometer measurements in drag-reducing channel flows. J Fluid Mech 70:369-392

Shen X, Kim K, Miller J, Sun Chee Fore R, Sirviente AI (2003) Experimental study of polymer drag reduction in a turbulent channel flow. In: Proceedings of the 4th ASME/JSME joint fluids engineering conference (FEDSM 2003), Honolulu, Hawaii, July 2003

Sun Chee Fore R, Szwalek JL, Sirviente AI (2004) The effects of polymer solution preparation and injection on drag reduction. J Fluids Eng (submitted)

Sureshkumar R, Beris AN, Handler RA (1997) Direct numerical simulation of the turbulent channel flow of a polymer solution. Phys Fluids 9:743-755

Tiederman WG, Luchick TS, Bogards DG (1985) Wall layer structure and drag reduction. J Fluid Mech 156:419-437

Virk PS (1975) Drag reduction fundamentals. AlCHE J 21:625-653

Vleggaar J, Tels M (1973) Drag reduction by polymer threads. Chem Eng Sci 28:965-968

Vlachogiannis M, Hanratty TJ (2004) Influence of wavy structured surfaces and polymer aggregation on drag reduction. Exp Fluids 36:685-700

Walker DT, Tiederman WG (1990) Turbulent structure in a channel flow with polymer injection at the wall. J Fluid Mech 218:377-403

Warhaft Z, Lumley JL (1978) An experimental study of decay of temperature fluctuations in grid-generated turbulence. J Fluid Mech 88:659-684

Warholic MD (1997) Modification of turbulent channel flow by passive and additive devices. PhD thesis, Chemical Engineering Department, University of Illinois at Urbana-Champaign, Illinois

Warholic MD, Massah H, Hanratty TJ (1999) Influence of drag-reducing polymers on turbulence: effects of Reynolds number, concentration and mixing. Exp Fluids 27:461-472

Wei T (1987) Reynolds number effects on the small-scale structure of a turbulent channel flow. PhD thesis, Department of Aerospace Engineering, University of Michigan, Michigan, USA

Wei T, Willmarth WW (1989) Reynolds number effects on the structure of a turbulent channel flow. J Fluid Mech 204:57-95

Wei T, Willmarth WW (1992) Modifying turbulent structure with drag-reducing polymer additives in turbulent channel flows. J Fluid Mech 245:619-641

Willmarth WW, Wei T, Lee CO (1987) Laser anemometer measurements of Reynolds stress in a turbulent channel flow with drag reducing polymer additives. Phys Fluids 30:933-935 